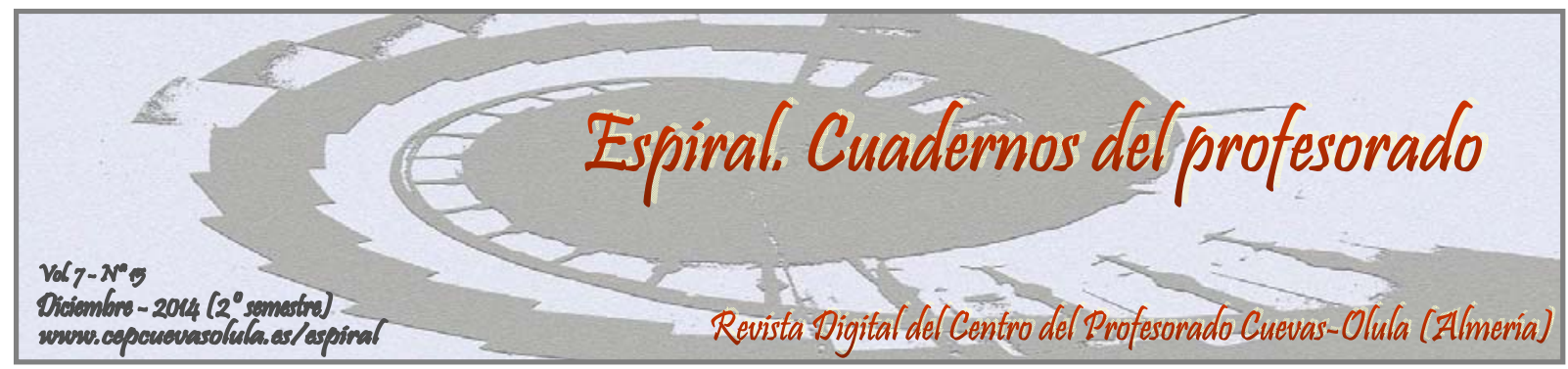

\title{
RESCATANDO LOS DEPORTES AUTÓCTONOS DE LA AMAZONIA PERUANA - PIRAGÜISMO / CANOTAJE
}

\section{RECOVERING THE INDIGENOUS SPORTS FROM THE PERUVIAN AMAZON - CANOE / RAFTING}

\section{Alessandra Maria Pinto Pereira}

Universidad de Murcia, España

RESUMEN: Este es un estudio práctico aplicado, criado por el Comité Olímpico Peruano (COPE) denominado Rescatando los Deportes Autóctonos de la Amazonia Peruana - Piragüismo / Canotaje, en colaboración con la Confederación Panamericana de Canotaje (COPAC), Confederación Sudamericana Canoe (CoSurCa), y la Facultad de Ciencias de la Actividad Física de la Universidad de Murcia, que tiene como objetivo introducir la modalidad deportiva en las poblaciones nativas de la Amazonia Peruana dándoles la oportunidad de practicar un deporte olímpico sin impacto ambiental, rescatar la práctica en canoa nativa, transmitir valores socio/educativos y todo lo que conlleva la práctica deportiva guiada. El estudio consta de varias fases cronológicas de ejecución, empezando en septiembre del 2012 con la I Etapa - Presentación del Piragüismo /Canotaje cómo deporte de competición, la Etapa II - Formación de Entrenadores y Participación en Competición, y la Etapa III - Expansión y Consolidación del Deporte. Actualmente el proyecto posee 14 deportistas participando en competiciones nacional e internacional, con 4 clubes deportivos y 25 entrenadores nivel I.

Palabras clave: Canotaje, Piragüismo, Amazonía, Perú.

ABSTRACT: This is a practical study, created by the Peruvian Olympic Committee, named "Recovering the Native Sports of the Peruvian Amazon - Canoeing and Kayaking", in cooperation with the Pan-American Canoe and Kayak Federation (COPAC), the South American Canoe and Kayak Federation (COSURCA) and the Faculty of Science of Physical Activity in Murcia. The purpose is to introduce this sport in the native population of the Peruvian Amazon and give them the opportunity to practice an Olympic sport without negative environmental impact, recover the practice of the native canoe and to transfer educational and social values. The study consists in different phases, starting with Phase I in September 2012 -Presentation of Canoeing and Kayaking as a Competition Sport, Phase II -Education of Coaches and Participation in Competition and Phase III - Expansion and Consolidation of the Sport. At the present, the project includes 14 athletes participating in national and international competitions, 4 canoe and kayak clubs and 25 level I coaches

Key words: Canoeing and kayaking, Amazon, Peru.

Pinto Pereira, A. M. (2014). Rescatando los deportes autóctonos de la amazonia Peruana piragüismo/canotaje. Espiral. Cuadernos del Profesorado, 7(15), 15-20. Disponible en: http://www.cepcuevasolula.es/espiral.

Fecha de recepción: 18/05/2014

Fecha de aceptación: 12/08/2014
Enviar correspondencia a: alessandrapinto@um.es 


\section{1.- INTRODUCCIÓN}

Desde siempre, el hombre se ha visto en la necesidad de utilizar embarcaciones para desplazarse por el agua con el impulso de buscar su alimento y su traslado, principalmente en las poblaciones ribereñas. Antiguamente esas embarcaciones eran fabricadas con restos de animales cómo pieles y huesos, así como de troncos de árboles, cortezas y vegetaciones cómo el junco o la totora.

Hoy en día las sofisticadas embarcaciones (Kayak y Canoa) hechas de fibra de carbono y otros materiales nos permiten recorrer un trayecto determinado con mayor velocidad, y con eso el origen de Deporte Olímpico desde 1936 conocido cómo Piragüismo o Canotaje que conocemos y disfrutamos en nuestros días (Rodríguez Inclán, 1993).

El Piragüismo / Canotaje es una modalidad deportiva que posibilita el desarrollo físico y la mejora de la salud, la transmisión de valores sociales, despierta una conciencia, el respeto medioambiental y la integración entre diversos grupos y etnias (Pinto Pereira, 2008).

\section{- El Deporte y la Salud}

Es de nuestro conocimiento que el deporte y la actividad física en general promocionan la salud, tanto previniendo como rehabilitando a las personas que lo practican. Además hay indicaciones importantes como que el deporte genera unos beneficios también de manera indirecta, siendo causante del rechazo de consumo de sustancias tóxicas (Van Veldhoven, 2014). Según los autores Casimiro y cols., 2001; Bergamaschi y cols., 2002; Nistal y cols., 2003; encontraron una relación entre la práctica físico deportiva y el descenso del consumo de sustancias toxicas como el tabaco, alcohol, drogas.

\section{- El Piragüismo / Canotaje y el Medio Ambiente}

Según autores cómo Luque, Baena-Extremera y Granero-Gallegos (2011), hay una sociedad preocupada por el entorno, por lo ecológico, lo rural, por el respeto al medio ambiente, en definitiva una sociedad que persigue el desarrollo sostenible. De acuerdo con la Comisión Mundial sobre el Medio Ambiente y Desarrollo de las Naciones Unidas: "El desarrollo sostenible es el desarrollo que satisface las necesidades del presente, sin comprometer la capacidad de las generaciones futuras para satisfacer sus propias necesidades” (Fullana y Ayuso, 2002, p. 27).

Es importante hacer saber que la práctica del piragüismo/canotaje se desarrolla exclusivamente en el medio natural, sin necesidad de talar árboles o contaminar el entorno con residuos de petróleo, no provoca ningún impacto medio ambiental. Entretanto, son los practicantes o deportistas los que deberán actuar como guardianes y conservadores del patrimonio natural para que sea un deporte sostenible y ecológico Macaya (2004).

\section{- El deporte y su influencia Social}

La actividad física y el deporte pueden considerarse como uno de los contextos más influyentes en la sociedad durante las últimas décadas. Siendo un marco muy propicio para la transmisión de valores y en el desarrollo tanto personal como social de los ciudadanos (Escartí, Pascual y Gutiérrez, 2005; Ruiz y Cabrera, 2004).

En ese sentido algunos autores como Cecchini, Montero, Alonso, Izquierdo y Contreras, 2007; Escartí, Buelga, Gutiérrez, y Pascual, 2009; Sánchez- Oliva, Leo, Sánchez-Miguel, Gómez y GarcíaCalvo (2011), afirman que la actividad física deportiva es un excelente medio de transmisión de valores educativos y culturales, tales como la cooperación, la empatía, la valoración del esfuerzo, los hábitos saludables, la autonomía o el respeto. Así como transmisor de ideologías, generador de economía, promotor de salud, medio de integración social y sobre todas ellas, destaca su función educadora.

Como transmisor de ideologías, el deporte es utilizado como símbolo de identificación local, deportiva o nacionalista, haciendo que muchas personas sientan como propios los mensajes y actuaciones que tiene su equipo (Vázquez 2001).

Con esto el Comité Olímpico Peruano (COPE) ha elaborado el proyecto denominado Rescatando los Deportes Autóctonos de la Amazonia Peruana - Piragüismo / Canotaje, en 
colaboración con la Confederación Panamericana de Canotaje (COPAC), Confederación Sudamericana Canoe (CoSurCa), y la Facultad de Ciencias de la Actividad Física de la Universidad de Murcia, que tiene como objetivo introducir la modalidad deportiva en las poblaciones nativas de la Amazonia Peruana dándoles la oportunidad de practicar un deporte olímpico sin impacto ambiental, rescatar la práctica en canoa nativa, transmitir valores socio/educativos y todo lo que conlleva la práctica deportiva guiada.

\section{2.- METODO}

El proyecto ya en desarrollo y consiste de diferentes fases (Figura 1), y son las siguientes:

- Etapa I - Presentación del Piragüismo /Canotaje cómo deporte de competición (2012/2013).

Esa Etapa se subdivide en dos fases:

- $\quad$ Fase I- empezó en septiembre del 2012, con la visita de miembros del COPE a las poblaciones de la selva Amazónica de Madre de Dios y Aguaytia, siendo el primer contacto con las autoridades y personas interesadas en llevar a cabo la constitución de un club, albergar las embarcaciones y realizar las sesiones de entrenamiento con los jóvenes.

- $\quad$ Fase II- En marzo del 2013, hemos visitado las poblaciones de la Selva Amazónica de Iquitos, Madre de Dios y el distrito del Callao - Lima. En esas visitas nos hemos reunido con más de 30 personas en cada pueblo, donde se impartió durante 8 horas una conferencia sobre el piragüismo/canotaje, explicando las embarcaciones utilizadas, la técnica de paleo, la enseñanza, las estrategias para constituir un club o escuela deportiva, captar a los jóvenes y formar líderes capaces de transmitir los valores propios del deporte.

\section{- Etapa II - Formación de Entrenadores y Participación en Competición}

- $\quad$ Formación de Entrenadores Lideres: En el mes de agosto del 2013, se realizó en la ciudad de Lima el I Curso de Entrenadores - Nivel I, a través de la Federación Internacional de Piragüismo, Solidaridad Olímpica y Comité Olímpico Peruano. Se reunieron 25 personas de diferentes zonas de Perú, y durante 10 días realizamos sesiones teóricas con siguientes contenidos: Introducción al deporte, tipos de embarcaciones, técnica de paleo, métodos de entrenamiento, planificación del entrenamiento, detección de talentos, nociones de nutrición, motivación y formación de líderes; y sesiones prácticas de paleo en kayak y en canoa. El curso fue clausurado con un examen teórico final para evaluar el nivel de aprendizaje y la entrega de los certificados.

- $\quad$ Participación en las Competiciones: En esta fase las poblaciones de Aguaytia y Madre de Dios, ya tuvieron su primera participación en el Campeonato Suramericano en Chile y en los Juegos Bolivarianos realizados en noviembre del 2013 en Perú con un equipo de 14 deportistas. Con esto el deporte ya comienza a crear unas bases sólidas para avanzar en las siguientes etapas.

- Etapa III - Expansión y consolidación del Piragüismo / Canotaje (2013-2014)

- $\quad$ El objetivo de esa fase es de constituir nuevos clubes y asociaciones deportivas por diferentes localidades de la Selva, cómo Pucallpa, Iquitos entre otras. Para eso hemos visitado la población Pucallpa y la laguna de Yarinacocha, con la intención de presentar a las autoridades y a la población local la modalidad del Piragüismo / canotaje, y en consecuencia constituir un Club deportivo dando la oportunidad a los jóvenes y a las comunidades nativas de practicar un deporte 
muy familiar para ellos, y consecuentemente transmitir los valores propios del deporte.

- $\quad$ Referente a la consolidación de la modalidad deportiva, nos hemos puesto en contacto con la Federación Peruana de Canotaje para establecer un Calendario Nacional y Regional de competiciones, de esta manera se definirán unos objetivos muy claros a nivel de deportistas, de clubes, y Federación Nacional e Internacional, determinando los campeones regionales, los nacionales y la participación en los eventos internacionales.

Etapa I - 2012/ 2013

Presentación del

Piragüismo /Canotaje a

la población nativa.

Figura 1. Etapas de la implantación del Proyecto - Rescatando los Deportes Autóctonos de la Amazonia Peruana - Piragüismo / Canotaje

\section{3.- CONSIDERACIONES FINALES}

Perú es un país con una extensión territorial de 1'285,216 Km2 de superficie; en la costa vive el 52,1\% de la población peruana, es decir, más de la mitad; la región andina alberga el 36,9 \%, en tanto que en el llano amazónico sólo vive el 11\% de la población total (Latinoamericano, \& de Demografía-CELADE, 2010).

Esas condiciones pueden ser consideradas un factor positivo para el desarrollo del Piragüismo/ Canotaje como un deporte de gran accesibilidad, pudiendo ser practicado en las playas del extenso litoral, en las diversas lagunas y en los ríos de la Selva Amazónica.

Sin embargo, hay también inconvenientes, cómo es el caso de Iquitos, que está situada en medio de la Amazonía y es considerada la ciudad más grande del mundo sin comunicación terrestre, para llegar se necesita 4 días de viaje en barco o en avión desde Lima a 1h30. Situación parecida hemos encontrado en Pucallpa y Aguaytia, que están situadas en el centro este del país, cruzando la Cordillera de los Andes por carreteras que muchas veces están en malas condiciones debido a los derrumbamientos ocasionados por las lluvias. 
Eso dificulta el logro de nuestros objetivos debido a los costosos desplazamientos de los equipos de deportistas para la participación en los eventos regionales y nacionales, los traslados de los entrenadores para asistir a cursos de formación, así como la entrega de las embarcaciones para la constitución de nuevos Clubes deportivos.

Entretanto somos consiente de que el simple hecho de salir a palear con una canoa no hace que la persona desarrolle hábitos saludables o que exista un cambio de valores sociales, apoyándonos en el estudio de Gómez (2005) que afirma: “ El deporte, por sí mismo, no transmite nada, es el docente el que, en último término, decide el valor pedagógico para la transmisión de valores de la práctica deportiva”, y en la tesis doctoral del Van Veldhoven (2014) basada en la Educación a través del Deporte, donde indica que: "Aquellos adolescentes que realizan una práctica físico-deportiva organizada, y sistemática suelen estar dirigidos por técnicos deportivos que les inculcan hábitos saludables y les alejan del consumo de sustancias tóxicas”.

Actualmente Perú cuenta con 14 deportistas masculinos y femeninos de las modalidades de Canoa y Kayak participando en selectivas nacionales y campeonatos internacionales, 4 Clubes deportivos (Puerto Maldonado, Aguaytía, Iquitos, y Callao), y 25 entrenadores nivel I - ICF listos para desarrollar sus funciones de Entrenadores y Educadores.

Por eso continuamos apoyando este novedoso proyecto de Rescatar el Canotaje en las comunidades nativas de la Amazonía Peruana, e introducir un deporte cómo una oportunidad de actividad física saludable, sin impacto medio ambiental y en la forma de expresar el orgullo Nacional.

\section{4.- REFERENCIAS}

Bergamaschi, A., Morri, M., Resi, D., Zanetti, F., y Stampi, S. (2002). Tobacco consumption and sports participation: a survey among university students in northern Italy. Annali de Ingieni, 14(5), 435-442.

Casimiro, A. J. (1999). Comparación, evolución y relación de hábitos saludables y nivel de condición física-salud en escolares, entre final de educación primaria (12 años) y final de educación secundaria obligatoria (16 años). Tesis Doctoral. Granada: Universidad de Granada.

Cecchini, J. A., Montero, J., Alonso, A., Izquierdo, M., y Contreras, O. (2007). Effects of per- sonal and social responsibility on fair play in sports and self-control in school-aged youths. European Journal of Sport Science, 7, 203-211.

Escartí, A., Buelga, F., Gutiérrez, M., y Pascual, C. (2009). El desarrollo positivo a través de la actividad física y el deporte: el programa de responsabilidad personal y social. Revista de psicología general y aplicada, 62(1-2), 45-52.

Fullana P. y Ayuso, S. (2002). Turismo sostenible. Rubes. Barcelona, España. pp. 27-30.

Gómez Rijo, A. (2005). La enseñanza y el aprendizaje de los valores en la educación Deportiva. Revista Internacional de Medicina y Ciencias de la Actividad Física y el Deporte, 5(18), 89-99.

Latinoamericano, C. y de Demografía-CELADE, C. (2010). Perú: estimaciones y proyecciones de población urbana y rural por sexo y edades quinquenales, según departamento, 2000-2015. Boletín especial, 19.

Luque, P., Baena-Extremera, A., y Granero-Gallegos, A. (2011). Buenas prácticas para un desarrollo sostenible en los eventos deportivos en el medio natural. Interciencia, 36(7), 531-537.

Macaya G (2004) La contribución del turismo y el deporte al desarrollo sostenible. Apunts: Educación Física y Deportes, 78, 51-55.

Nistal, P., Prieto, J. A., Del Valle, M., y González, V. (2003). Relación de la actividad física con el consumo de tabaco en adolescentes. Archivos de Medicina del Deporte, 20(97), 397-403.

Pinto Pereira, A. M. (2008). Análisis isocinético de la fuerza de la musculatura del hombro en piragüistas de élite juvenil - Tesis Doctoral. Disponible en: digitum.um.es

Rodríguez Inclán, J. (1993). Historia del Piragüismo. Comité Olímpico Español: Piragüismo Vol. I. Madrid.

Ruiz, G. y Cabrera, D. (2004). Los valores en el deporte. Revista de Educación, 335, 9-19.

Sánchez-Oliva, D., Leo, F. M., Sánchez-Miguel, P. A., Gómez, F. R., y García-Calvo, T. (2011). Teoría de autodeterminación y comportamientos prosociales en jóvenes jugadores de fútbol. Apunts: Educación física y deportes, 108(1), 32-38. 
Van Veldhoven, L. H. M. (2014). Condición física saludable y hábito de consumo de tabaco en adolescentes escolarizados de la Región de Murcia. Proyecto de investigación: digitum.um.es

Vázquez, B. (2001). Deporte y educación. En B. Vázquez (coord.), O. Camerino, M. González, F. del Villar, J. Devís y P. Sosa. Bases educativas de la actividad física y el deporte (pp. 333-355). Madrid: Síntesis.

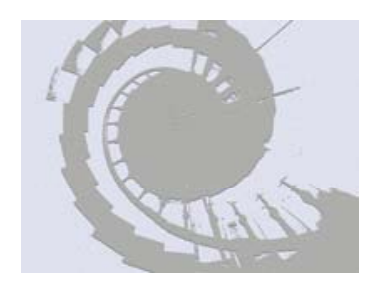

